\title{
Zur Theorie der Absorption von Gasansammlungen im Organismus unter besonderer Berücksidhtigung der Atelektase-Bildung
}

\author{
Von Hans H. Loeschcke \\ Aus dem Physiologischen Institut der Universität Göttingen \\ (Z. Naturforschg. 13 b, 803-811 [1958]; eingegangen am 12. Juli 1958)
}

\begin{abstract}
Es werden Modellvorstellungen entwickelt, die zum Verständnis der Absorption von Gasansammlungen im Organismus beitragen. Mit ihrer Hilfe wurde als Beispiel die „stationäre“ Zusammensetzung des Gases in einem durch Verschluß des Bronchus während der Atmung von Luft von der Außenluft abgetrennten Lungenabschnittes unter vereinfachenden Annahmen berechnet. Es ergibt sich, daß $\mathrm{CO}_{2}$ - und $\mathrm{O}_{2}$-Druck in der Gasphase um weniger als 0,1 Torr höher zu erwarten sind als im gemischten venösen Blut. Die $\mathrm{N}_{2}$-Differenz ist größer. Sie ist bestimmend für die AbsorptionsGeschwindigkeit. Eine Prüfung von Rechnung und Approximationen wird durchgeführt durch Vergleich der berechneten Gas-Diffusionsströme mit experimentellen Daten der Literatur und durch Berechnung des Diffusionsfaktors (diffusion capacity) der Lunge aus berechnetem Diffusionsstrom und berechneten mittleren Partialdruck-Differenzen. Die Übereinstimmung ist befriedigend. Ein Grund für die Annahme außer einfacher Diffusion wirkender anderer Prozesse bei der Entstehung von Atelektasen der Lunge nach Bronchialverschluß besteht nicht.
\end{abstract}

\section{1. "Grenzschicht-Modell“" der Gasabsorption}

In früheren Arbeiten ${ }^{1,2}$ wurde die Gasabsorption in einem Modell besprochen, in dem eine Gasansammlung angenommen war, die von einer bewegten Flüssigkeit durch eine unbewegte Grenzschicht getrennt war. Es war dort angenommen worden, daß die bewegte Flüssigkeit unbegrenzt sei und daß die Konvektion so groß sei, daß in der bewegten Flüssigkeit überall die gleichen Gasdrucke herrschten. Besteht in der bewegten Flüssigkeit ein geringerer Gesamtgasdruck als in der Gasphase, so bildet sich ein Diffusionsgefälle für die einzelnen Gase durch die unbewegte Grenzschicht aus. In der Gasblase stellt sich ein „stationärer Zustand“ (RIst und Strohl ${ }^{3}$ bzw. Jost ${ }^{4}$ ) ein, der durch Konstanz der Partialdrucke der einzelnen Gase gekennzeichnet ist. Von DALE und RAHN ${ }^{5}$ wurde dieser Zustand „state of constant composition“ genannt.

Er ist dadurch gekennzeichnet, daß die partiellen Diffusionsströme der einzelnen Gase $\dot{V}_{\mathrm{i}}$ den Konzentrationen dieser Gase (oder ihren Partialdrucken $\left.P_{\mathrm{i}}\right)$ proportional sind:

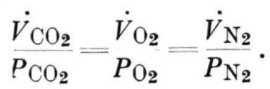

Die Zusammensetzung des Gases kann dann durch Einsetzen des 1. Fi ck schen Gesetzes

$$
\dot{V}_{\mathrm{i}}=\alpha_{\mathrm{i}} D_{\mathrm{i}} \frac{F}{d} \Delta P_{\mathrm{i}}
$$

1 H. H. Loeschcke, Klin. Wschr. 34, 801 [1956].

2 H. H. Loeschcke, Z. Naturforschg. 11 b, 613 [1956].

3 E. Rist u. A. Strohl, Presse méd. 1922, 69. berechnet werden, wobei $F / d$ aus dem Gleichungssystem herausfällt.

$$
\begin{aligned}
\alpha_{\mathrm{CO}_{2}} D_{\mathrm{CO}_{2}} \frac{\Delta P_{\mathrm{CO}_{2}}}{P_{\mathrm{CO}_{2}}}=\alpha_{\mathrm{O}_{2}} D_{\mathrm{O}_{2}} & \frac{\Delta P_{\mathrm{O}_{2}}}{P_{\mathrm{O}_{2}}}= \\
& =\alpha_{\mathrm{N}_{2}} D_{\mathrm{N}_{2}} \frac{\Delta P_{\mathrm{N}_{2}}}{P_{\mathrm{N}_{2}}}=u,
\end{aligned}
$$

wobei $u=\frac{\dot{V}_{\mathrm{i}} d}{F P_{\mathrm{i}}}$ ist. Die Symbole bedeuten:

$\alpha=$ Bunsenscher Absorptionskoeffizient $\left(\right.$ at $\left.^{-1}\right)$,

$D=$ Diffusionskoeffizient $\left(\mathrm{cm}^{2} \mathrm{sec}^{-1}\right)$,

$\Delta P_{\mathrm{i}}=$ Partialdruck-Gefälle eines Gases i zwischen Gasund Flüssigkeitsphase (at),

$P_{\mathrm{i}}=$ Partialdruck des gleichen Gases in der Gasphase (at),

$F \quad=$ Austauschfläche $\left(\mathrm{cm}^{2}\right)$,

$d=$ „Diffusions-Schichtdicke“ $(\mathrm{cm})$.

Für die Berechnung wird noch eingesetzt, daß

ist.

$$
P_{\mathrm{CO}_{2}}+P_{\mathrm{O}_{2}}+P_{\mathrm{N}_{2}}=B-P_{\mathrm{H}_{2} \mathrm{O}}
$$

Berechnungen eines solchen physikalischen Modells sind von Jost $^{4}$ und von LoEschскE ${ }^{2}$ für bestimmte Bedingungen gegeben worden, worauf an dieser Stelle verwiesen werden kann. Sind die Partialdrucke in Flüssigkeit und Gasphase bekannt, so kann auch die Geschwindigkeit der Absorption unter Benutzung des 1. Fickschen Gesetzes (Gl. 2) erhalten werden ${ }^{2,4}$. Berechnungen dieser Art sind für den Fall des Pneumothorax wenigstens in der Größenordnung in Übereinstimmung mit den experimen-

4 W. Jost, Z. Naturforschg. 4 b, 318 [1949].

5 W. A. Dale u. H. RahN, Amer. J. Physiol. 170, 606 [1952]. 
tellen Befunden. Berechnet man aber auf diese Art die Absorptionszeiten von Atelektasen, so erhält man sehr viel kürzere Zeiten als experimentell gefunden wurden (Lichtheim ${ }^{6}$, Coryllos und Birnbaum ${ }^{7}$ und $D_{A L E}$ und $\left.R_{A h N}{ }^{5}\right)$. Der Grund für diese ungenügende Übereinstimmung könnte in Folgendem gesucht werden.

\section{Modell mit Berücksichtigung des Gas- transports durch den Blutkreislauf}

Eine Gasansammlung im Organismus, sei es ein Pneumothorax, Pneumoperitoneum, eine subcutane Gasblase oder Alveolargas in einem Bronchialgebiet, dessen zuführender Bronchus verschlossen ist, unterscheidet sich in einem Punkt grundsätzlich von dem beschriebenen physikalischen Modell. Die bewegte Flüssigkeit ist hier ersetzt durch das strömende Blut in den der Gasphase benachbarten Blutgefäßen. Diese bewegte Flüssigkeitsphase ist nicht unbegrenzt und es kann nicht angenommen werden, daß die Gaspartialdrucke in ihr konstant seien. Vielmehr gleichen sich die Gas-Partialdrucke im Blut, während dieses an der Gasblase vorbeiströmt, mehr oder weniger an die Gas-Partialdrucke in der Blase an, und man stößt hier auf Probleme, wie sie auch bei der Betrachtung des normalen Gasaustausches in der Lunge auftreten.

Es soll in dieser Arbeit versucht werden, dem „Grenzschichtmodell“ der Gasabsorption ein mehr den biologischen Gegebenheiten angepaßtes Modell gegenüberzustellen, das sich von dem ersteren dadurch unterscheidet, daß die Änderungen der GasPartialdrucke in dem an der Gasphase vorbeiströmenden Blut auf der Austauschstrecke berücksichtigt werden.

In Abb. 1 ist schematisch der Ausgleich des Partialdruckes für irgendein Gas mit einer die Gasblase auf der Strecke $l_{\mathrm{a}}$ berührenden Blutkapillare gezeichnet, in die das Blut mit dem Gas-Partialdruck ${ }^{0} P_{\mathrm{i}}$ eintritt und mit dem Partialdruck $P_{\mathrm{i}}{ }^{\prime}$ austritt. Der Partialdruck in der Gasphase sei $P_{\mathrm{i}}$ (i steht für $\mathrm{CO}_{2}, \mathrm{O}_{2}$ oder $\mathrm{N}_{2}$ ), und die Ausgangs- und Enddifferenzen des Partialdrucks zwischen Gasphase und Blut seien $\left(\Delta P_{0}\right)_{\mathrm{i}}$ und $\left(\Delta P^{\prime}\right)_{\mathrm{i}}$. Die mittlere Partial-

6 Lichtheim, Naunyn-Schmiedebergs Arch. exp. Pathol. Pharmakol. 10, 54 [1879].

7 P. N. Coryllos u. G. L. Birnbaum, Arch. Surgery 21, 1214 [1930].

* Es muß bemerkt werden, daß in dem üblichen Verfahren der Integration erhebliche Approximationen stecken, ins-
druck-Differenz sei $\Delta P_{\mathrm{i}}$ und so gemittelt, daß Einsetzen von $\Delta P_{\mathrm{i}}$ in die Diffusions-Gleichung den Diffusionsstrom richtig wiedergibt.

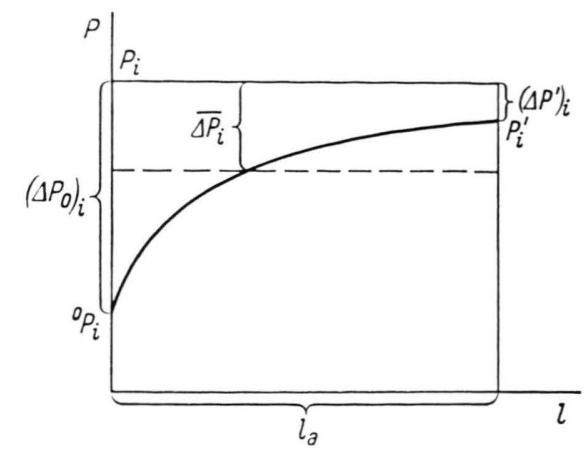

Abb. 1. Schema der Angleichung des Partialdruckes eines Gases im Blut $\left(\mathrm{O}_{2}\right)$ an die Alveolarluft auf der Strecke der Lungenkapillare. Erklärung der Bezeichnungen im Text.

In Wirklichkeit findet der Gasaustausch nicht mit einem einzigen Blutgefäß, sondern mit einer Population von solchen Gefäßen statt. Aber in jedem Fall kann ein Mittelwert der einzelnen $\overline{\Delta P}$ gedacht werden, der den Diffusionsstrom eines Gases aus der Gasphase in das Blut richtig wiedergibt. Er sei als Mittelwert von Mittelwerten mit $\overline{\Delta P}$ bezeichnet.

Die mittlere Partialdruck-Differenz $\overline{\Delta P}$ ist identisch mit dem für die Betrachtung des Gasaustausches in der Lunge allgemein verwendeten, durch die B o h r sche Integration erhaltenen mittleren Partialdruck-Differenz, die für inerte Gase einfach berechnet werden kann, wenn der Partialdruck des Gases in der Gasphase und der Anfangs- und Endpartialdruck in der Blutphase bekannt sind, während für Gase, die chemisch gebunden sind wie $\mathrm{O}_{2}$ und $\mathrm{CO}_{2}$ ein graphisches Verfahren zu seiner Bestimmung notwendig ist *.

Es ist nun offensichtlich so, daß $\overline{\Delta P}$ um so mehr von $\Delta P_{0}$ abweicht, und zwar kleiner ist als $\Delta P_{0}$, je schneller der Ausgleich der Partialdrucke zwischen Blut und Gasphase stattfindet. Die chemische Bindung von $\mathrm{CO}_{2}$ und $\mathrm{O}_{2}$ wirkt in der Richtung, daß sie die Angleichung verzögert und dadurch das Verhältnis $\overline{\Delta P} / \Delta P_{0}$ größer sein läßt, als es bei gleichem $\triangle P_{0}$ und gleichem Diffusions-Koeffizienten und gleicher Löslichkeit sein würde.

besondere daß die „Längsdiffusion" nicht berücksichtigt ist. Es wird hier auf Thews ${ }^{8,9}$ verwiesen.

8 G. Thews, Acta biotheoretica 10, 105 [1953].

9 G. Thews, Pflügers Arch. ges. Physiol. Menschen, Tiere 265, 154. [1957]. 
Die Gl. (1) für den Zustand konstanter Zusammensetzung gilt offensichtlich auch für eine Gasansammlung, die im Austausch mit durchbluteten Gefäßen steht, wenn man statt $\Delta P_{\mathrm{i}} \overline{\overline{\Delta P}}_{\mathrm{i}}$ einsetzt:

$$
\begin{aligned}
& a_{\mathrm{CO}_{2}} D_{\mathrm{CO}_{2}} \frac{\overline{\Delta P}_{\mathrm{CO}_{2}}}{P_{\mathrm{CO}_{2}}}=\alpha_{\mathrm{O}_{2}} D_{\mathrm{O}_{2}} \frac{\overline{\Delta P_{\mathrm{O}_{2}}}}{P_{\mathrm{O}_{2}}}= \\
&=\alpha_{\mathrm{N}_{2}} D_{\mathrm{N}_{2}} \frac{\overline{\Delta P}_{\mathrm{N}_{2}}}{P_{\mathrm{N}_{2}}}=u .
\end{aligned}
$$

Dies bedeutet, daß die Zusammensetzung der Gase in einer Gasblase, die in durchblutetem Gewebe liegt, anders sein muß als die für das „Grenzschichtmodell" berechnete ${ }^{2}$. Gl. (4) entspricht Gl. (3) am physikalischen Modell. Im Gegensatz zu Gl. (3) erlaubt sie noch keine Berechnung der Zusammensetzung der Gasphase, wenn die Partialdrucke in der Blutphase und die Summe der Partialdrucke in der Gasphase bekannt sind, da die Mittelwertbildung $\overline{\Delta P}$ neue unbekannte Größen einführt.

Von RaHN und van Liew ${ }^{10}$ wurde ein anderer Weg vorgeschlagen, der ebenfalls von der Gl. (1) für den Zustand konstanter Zusammensetzung ausgeht, aber den Diffusionsstrom der einzelnen Gase nicht durch das Diffusionsgesetz, sondern durch das F i c k sche Prinzip ausdrückt.

Der Diffusionsstrom eines Gases i, $\dot{V}_{i}$, ist dann in folgender Weise einzusetzen:

$$
\dot{V}_{\mathrm{i}}=\dot{V}_{\mathrm{bl}} \cdot \beta_{\mathrm{i}}\left(P_{\mathrm{i}}{ }^{\prime}-{ }^{0} P_{\mathrm{i}}\right),
$$

wenn $\dot{V}_{\mathrm{bl}}$ die Stromstärke $\left(\mathrm{cm}^{3} \mathrm{~min}^{-1}\right)$ des an der Gasblase vorbeifließenden Blutes, $\beta_{\mathrm{i}}$ die „scheinbare Löslichkeit“, d. h. das bei Änderung des Partialdruckes um eine Einheit von der Volumeneinheit des Blutes aufgenommene Volumen des Gases i unter Standardbedingungen, $P_{\mathrm{i}}$ den Partialdruck des Gases i am stromaufwärtigen und $P_{\mathrm{i}}^{\prime}$ den Partialdruck des Gases a am stromabwärtigen Ende des mit dem Gase in Kontakt stehenden Blutgefäßes darstellt.

Aus (1) wird dann:

$$
\begin{aligned}
\beta_{\mathrm{CO}_{2}} \frac{P^{\prime} \mathrm{CO}_{2}-{ }^{0} P_{\mathrm{CO}_{2}}}{P_{\mathrm{CO}_{2}}}=\beta_{\mathrm{O}_{2}} & \frac{P^{\prime} \mathrm{O}_{2}-{ }^{0} P_{\mathrm{O}_{2}}}{P_{\mathrm{O}_{2}}}= \\
& =\beta_{\mathrm{N}_{2}} \frac{P^{\prime} \mathrm{N}_{2}-{ }^{0} P_{\mathrm{N}_{2}}}{P_{\mathrm{N}_{2}}}=v,
\end{aligned}
$$

wobei $v=\frac{\dot{V}_{\mathrm{i}}}{\dot{V}_{\mathrm{bl}} \cdot P_{\mathrm{i}}}$ ist.

10 H. Rahn u. H. D. van Liew, Wright Air Development Command Tech. Report 55-357, 382 [1955].
Berechnung der stationären Zusammensetzung der Gasphase ist auch mit (6 a) nicht möglich ohne zusätzliche Annahme über die drei neuen Unbekannten $P_{\mathrm{i}}^{\prime}$. Aussagen hierüber sind nicht ohne Zuhilfenahme der Diffusionsgesetze möglich. Dies kann aber geschehen durch Kombination von (6a) mit (4), wobei man erhält:

$$
\begin{aligned}
& \frac{\alpha_{\mathrm{CO}_{2}} D_{\mathrm{CO}_{2}}}{\beta_{\mathrm{CO}_{2}}} \frac{\overline{\Delta P_{\mathrm{CO}_{2}}}}{P^{\prime} \mathrm{CO}_{2}-{ }^{0} P_{\mathrm{CO}_{2}}}=\frac{\alpha_{\mathrm{O}_{2}} D_{\mathrm{O}_{2}}}{\beta_{\mathrm{O}_{2}}} \frac{\overline{\Delta P_{\mathrm{O}_{2}}}}{P^{\prime} \mathrm{O}_{2}-{ }^{0} P_{\mathrm{O}_{2}}}= \\
& =\frac{\alpha_{\mathrm{N}_{2}} D_{\mathrm{N}_{2}}}{\beta_{\mathrm{N}_{2}}} \frac{\overline{\Delta P}_{\mathrm{N}_{2}}}{P^{\prime} \mathrm{N}_{2}-{ }^{0} P_{\mathrm{N}_{2}}}=\frac{u}{v}, \quad \frac{u}{v}=\frac{d}{F} \cdot \dot{V}_{\mathrm{bl}} .
\end{aligned}
$$

Ehe dieser Weg weiter verfolgt werden kann, ist eine Untersuchung der Beziehung zwischen $\overline{\overline{\Delta P_{\mathrm{i}}}}$ und $P_{\mathrm{i}}^{\prime}-{ }^{0} P_{\mathrm{i}}$ notwendig.

\section{Die Angleichung der Gas-Partialdrucke im Blut an die Partialdrucke in der Gasphase. \\ Die mittleren Partialdruck-Differenzen zwischen Gasphase und Blut}

Nimmt man ein Blutgefäß in Kontakt mit der Gasphase an, so nimmt die Partialdruck-Differenz eines Gases $\Delta P$ von einem Ausgangswert $\Delta P_{0}$ bis auf einen Endwert von $\Delta P^{\prime}$ am Ende des Blutgefäßes von der Länge $l_{\mathrm{a}} \mathrm{ab}(\mathrm{Abb} .1)$. In jeder Stelle ist der Gasstrom $\dot{V}_{\mathrm{i}}$ dem Partialdruck-Gefälle $\Delta P_{\mathrm{i}}$ an der gleichen Stelle proportional. Ist nun der Anstieg des Gas-Partialdruckes in der Flüssigkeit auf der Wegstrecke $d l$ der Menge des auf dieser Strecke einströmenden Gases proportional, was streng nur bei einem nicht chemisch gebundenen Gas der Fall ist, so ist auch der Anstieg des Partialdruckes im Blut (gleich der Abnahme des Gefälles - $d \Delta P$ ) auf dem Wege $d l$ der örtlichen Partialdruck-Differenz $\Delta P$ proportional.

$$
\frac{d \Delta P}{d l}=k \cdot \Delta P
$$

und durch Integration über $l$ :

$$
\ln \frac{\Delta P}{\Delta P_{0}}=-k l
$$

oder

$$
\Delta P=\Delta P_{0} \cdot e^{-k l * *} .
$$

\footnotetext{
** Eine ausführliche Ableitung dieser Funktion ergibt: $\Delta P=\Delta P_{0} \cdot \exp \left(-\alpha D F_{\mathrm{a}} l / \beta d \dot{V} l_{\mathrm{a}}\right) \quad\left(F_{\mathrm{a}}=\right.$ Austauschfläche $)$. Das stimmt mit der Formel von BoHr ${ }^{11}$ überein.
} 
Um aus dieser Funktion $\Delta P$ zu erhalten, ist Integration von $(8 \mathrm{~b})$ über die Austauschstrecke $l_{\mathrm{a}}$ notwendig, da $\Delta P$ zu definieren ist als:

$$
\begin{aligned}
\overline{\Delta P} & \equiv \frac{\int_{0}^{l_{a}} \Delta P d l}{l_{\mathrm{a}}}, \\
\int_{0}^{l_{a}} \Delta P d l & =\int_{0}^{l_{a}} \Delta P_{0} \cdot e^{-k l} \cdot d l, \\
\int_{0}^{l_{a}} \Delta P d l & =-\Delta P_{0} \frac{e^{-k l_{\mathrm{a}}}}{k}+c .
\end{aligned}
$$

Setzt $\operatorname{man} l=0$, so ergibt sich:

$$
\begin{gathered}
c=\Delta P_{0} \frac{1}{k}, \\
\int_{0}^{l_{a}} \Delta P d l=-\Delta P_{0} \frac{e^{-k l_{\mathrm{a}}}}{k}+\frac{\Delta P_{0}}{k}, \\
\int_{0}^{l_{a} \Delta P d l}=\Delta P=\frac{\Delta P_{0}}{k l_{\mathrm{a}}}\left(1-e^{\left.-k l_{\mathrm{a}}\right),}\right. \\
\frac{\Delta P}{\Delta P_{0}}=\frac{l-e^{-k l_{\mathrm{a}}}}{k l_{\mathrm{a}}} .
\end{gathered}
$$

$k$ wird aus (8 a) unter Einsetzung von $\Delta P=\Delta P^{\prime}$ und $l=l_{\mathrm{a}}$ erhalten als:

$$
k=\frac{\ln \left(\Delta P_{0} / \Delta P^{\prime}\right)}{l_{\mathrm{a}}} .
$$

Setzt man (10) ein in (9), so kommt

$$
\overline{\Delta P}=\frac{\Delta P_{0}-\Delta P^{\prime}}{\ln \left(\Delta P_{0} / \Delta P^{\prime}\right)}
$$

oder da $\Delta P_{0}-\Delta P^{\prime}=P^{\prime}-{ }^{0} P$,

$$
\overline{\Delta P}=\frac{P^{\prime}-{ }^{0} P}{\ln \left(\Delta P_{0} / \Delta P^{\prime}\right)} .
$$

Setzt man (10) ein in (4), so erhält man folgende Formel:

$$
\begin{gathered}
\frac{\beta_{\mathrm{CO}_{2}}}{\alpha_{\mathrm{CO}_{2}} D_{\mathrm{CO}_{2}}} \ln \left(\frac{\Delta P_{0}}{\Delta P^{\prime}}\right)_{\mathrm{CO}_{2}}=\frac{\beta_{\mathrm{O}_{2}}}{\alpha_{\mathrm{O}_{2}} D_{\mathrm{O}_{2}}} \ln \left(\frac{\Delta P_{0}}{\Delta P^{\prime}}\right)_{0_{2}}= \\
=\frac{\beta_{\mathrm{N}_{2}}}{\alpha_{\mathrm{N}_{2}} D_{\mathrm{N}_{2}}} \ln \left(\frac{\Delta P_{0}}{\Delta P^{\prime}}\right)_{\mathrm{N}_{2}}=\frac{v}{\mathrm{u}} .
\end{gathered}
$$

Diese Gleichung ist nicht mehr charakteristisch für den stationären Zustand, sondern allgemein gültig. Sie kann auch für jedes Gas einzeln erhalten werden durch Kombination von (2), (5) und (11). Sie erlaubt den Vergleich des Verlaufes der Angleichung des Partialdruckes zweier Gase im Blute auf der Kontaktstrecke mit der Gasphase. Es muß aber

11 H. Rahn u. E. Canfield, Wright Air Development Command Tech. Report 55-357, 395 [1955]. sehr deutlich betont werden, daß dies exakt nur dann möglich ist, wenn der Partialdruck aller Gase im Blut linear mit dem Gaseinstrom ansteigt, und wenn auch nicht Wechselwirkungen zwischen zwei Gasen verschiedener Austauschgeschwindigkeiten auftreten. Hierdurch ist die Anwendbarkeit begrenzt auf diejenigen Fälle, in denen die Bindungskurven als linear in dem vorkommenden Bereich der Partialdrucke angesehen werden können. Die Tatsache der Wechselwirkung zwischen $\mathrm{CO}_{2}$ - und $\mathrm{O}_{2}$-Bindung im Blut (B o h r - Effekt) würde eigentlich die Verwendung von (12) für beide Gase verbieten. Da aber Gl. (12) nur verwendet werden soll, um Schlüsse über die Größenordnung der Austausch-Unterschiede zu ziehen, schien die Verwendung der Gleichung in diesem Falle erlaubt.

\section{Wahl der Atelektase-Bildung bei Bronchialverschluß als Beispiel}

Aus den eben diskutierten Gründen ist es an dieser Stelle notwendig, sich für die weitere Diskussion auf ein bestimmtes Beispiel zu beschränken. Es soll im folgenden darum von der Atelektase-Bildung (Gasabsorption) in einem durch Bronchialverschluß abgeschlossenen Lungenabschnitt bei Luftatmung gesprochen werden. Das Atelektase-Gebiet soll klein sein gegenüber der freibeatmeten Lunge, so daß die Gas-Partialdrucke im venösen Mischblut durch den Gasaustausch im Atelektase-Gebiet nicht beeinflußt werden. Dies Beispiel hat zwei Vorteile. Erstens ist damit zu rechnen, daß der Gasaustausch in allen Kapillaren in annähernd gleicher Weise vor sich geht, so daß die Mittelung über viele Kapillaren sich erübrigt, und zweitens, daß man sich im Bereiche des angenähert geraden Teils der Sauerstoffbindungs-Kurve befindet, da venöses Mischblut in das Atelektase-Gebiet einfließt und der Sauerstoffdruck in der Gasphase nicht weit vom Sauerstoffdruck des venösen Mischbluts entfernt sein kann. Schließlich liegen neuere experimentelle Befunde über die Gasabsorption in der abgeschlossenen Lunge vor (DALE und $R_{A H N}{ }^{5}$ ), die einen Vergleich der Rechnung mit dem experimentellen Ergebnis erlauben. Auf Untersuchungen der Gasabsorption an subkutanen Gasdepots durch RaHN und Canfield ${ }^{11}$ und durch van LIEW ${ }^{12}$ sowie auf die theoretische

12 H. D. van Liew, Wright Air Development Command Tech. Report 55-357, 409 [1955]. 
Arbeit von RaHN und van Liew ${ }^{10}$ soll an dieser Stelle verwiesen werden. RaHn ${ }^{13}$ hat seine Ergebnisse neuerdings zusammengefaßt.

Demonstration der Vollständigkeit des PartialdruckAusgleichs am Ende der Kapillare für $\mathrm{CO}_{2}$ und $\mathrm{N}_{2}$

Gl. (12) kann in die beiden folgenden Gln. aufgelöst werden:

$$
\left(\frac{\Delta P_{0}}{\Delta P^{\prime}}\right)_{\mathrm{CO}_{2}}=\left(\frac{\Delta P_{0}}{\Delta P^{\prime}}\right)_{\mathrm{O}_{2}} \frac{\beta_{\mathrm{O}_{2}} \alpha_{\mathrm{CO}_{2}} D_{\mathrm{CO}_{2}}}{\beta_{\mathrm{CO}_{2} \alpha_{\mathrm{O}_{2}} D_{\mathrm{O}_{2}}}}
$$

und $\quad\left(\frac{\Delta P_{0}}{\Delta P^{\prime}}\right)_{\mathrm{N}_{2}}=\left(\frac{\Delta P_{0}}{\Delta P^{\prime}}\right)_{\mathrm{O}_{2}} \frac{\beta_{\mathrm{O}_{2} \alpha_{\mathrm{N}_{2}} D_{\mathrm{N}_{2}}}}{\beta_{\mathrm{N}_{2} \alpha_{\mathrm{O}_{2}} D_{\mathrm{O}_{2}}}}$.

Nimmt man willkürlich an, daß der Druckausgleich für $\mathrm{O}_{2}$ nur bis zur Hälfte des vollständigen Ausgleichs gehe, also:

$$
\left(\frac{\Delta P_{0}}{\Delta P^{\prime}}\right)_{\mathrm{O}_{2}} \equiv 2
$$

dann erhält man, wenn man die in Tab. 1 zusammengestellten Werte für $\alpha, \beta$ und $D$ einsetzt,

\begin{tabular}{|c|c|l|c|}
\hline & $\alpha_{\mathrm{at}^{-1}}^{*}$ & $\beta_{\mathrm{at}^{-1}}$ & $\begin{array}{c}\mathrm{D} * * \\
10^{-5} \mathrm{~cm}^{2} \mathrm{sec}^{-1}\end{array}$ \\
\hline $\mathrm{CO}_{2}$ & 0,567 & 2,3 & 3,13 \\
$\mathrm{O}_{2}$ & 0,024 & 1,5 & 3,21 \\
$\mathrm{~N}_{2}$ & 0,012 & 0,012 & 3,01 \\
\hline
\end{tabular}

Tab. 1. Die verwendeten Werte für den B u n s e n schen Absorptionskoeffizienten $\alpha$, die ,scheinbare Löslichkeit“ $\beta$ und den Diffusionskoeffizienten $D$ für die Gase $\mathrm{CO}_{2}, \mathrm{O}_{2}$ und $\mathrm{N}_{2}$.

* Werte für Wasser nach Handbook of Chemistry and Physics. ** Werte für Wasser nach Gertz und Loeschcke ${ }^{15}$.

folgende Zahlenwerte für $\Delta P_{0} / \Delta P^{\prime}$ der Gase $\mathrm{CO}_{2}$ und $\mathrm{N}_{2}$ :

$$
\begin{aligned}
& \left(\frac{\Delta P_{0}}{\Delta P^{\prime}}\right)_{\mathrm{CO}_{2}}=2^{15}=3,3 \cdot 10^{4}, \\
& \left(\frac{\Delta P_{0}}{\Delta P^{\prime}}\right)_{\mathrm{N}_{2}}=2^{58,5}=3,2 \cdot 10^{17}
\end{aligned}
$$

oder allgemeiner, wenn man $\left(\frac{\Delta P_{0}}{\Delta P^{\prime}}\right)_{\mathrm{O}_{2}}=q$ setzt:

$$
\left(\frac{\Delta P_{0}}{\Delta P^{\prime}}\right)_{\mathrm{CO}_{2}}=q^{15} \quad \text { und }\left(\frac{\Delta P_{0}}{\Delta P^{\prime}}\right)_{\mathrm{N}_{2}}=q^{58,5} \text {. }
$$

Auch wenn man die eingesetzten Zahlenwerte in mäßigem Grade variiert, bleibt bestehen, daß man hohe Exponenten erhält. Es ergibt sich, daß selbst für hohe Ansprüche an die Genauigkeit die Endgradienten $\triangle P^{\prime}$ für $\mathrm{CO}_{2}$ und $\mathrm{N}_{2}$ gegenüber den Anfangsgradienten $\Delta P_{0}$ zu vernachlässigen sind. Es kann dann für den Partialdruck am Ende der Kapillare $P^{\prime}$ für diese beiden Gase der Partialdruck

13 H. RaHN, Fed. Proc. 16, 685 [1957].

14 Сн. Boнr, Skand. Arch. Physiol. 22, 221 [1909]. in der Gasphase $P$ eingesetzt werden. Damit verschwinden zwei von den drei Unbekannten $P_{\mathrm{i}}^{\prime}$ in Gl. (6). Für plausible Annahmen über $P^{\prime}{ }_{2}$ ist es nun möglich, die Zusammensetzung des AtelektaseGases zu berechnen.

\section{Berechnung der stationären Gaszusammensetzung im Atelektase-Gebiet}

Gl. (6) kann nun in folgender Weise geschrieben werden :

$$
\begin{aligned}
\beta_{\mathrm{CO}_{2}} \frac{P_{\mathrm{CO}_{2}}-{ }^{0} P_{\mathrm{CO}_{2}}}{P_{\mathrm{CO}_{2}}}=\beta_{\mathrm{O}_{2}} \frac{P^{\prime} \mathrm{O}_{2}-{ }^{0} P_{\mathrm{O}_{2}}}{P_{\mathrm{O}_{2}}}= \\
=\beta_{\mathrm{N}_{2}} \frac{P_{\mathrm{N}_{2}}-{ }^{0} P_{\mathrm{N}_{2}}}{P_{\mathrm{N}_{2}}} .
\end{aligned}
$$

Werden für ${ }^{0} \mathrm{P}_{\mathrm{CO}_{2}},{ }^{0} \mathrm{P}_{\mathrm{O}_{2}}$ und ${ }^{0} \mathrm{P}_{\mathrm{N}_{2}}$ Normalwerte des venösen Mischbluts eingesetzt und für $P_{\mathrm{O}_{2}}^{\prime}$ bzw. $\left(\Delta P_{0} / \Delta P^{\prime}\right)_{\mathrm{O}_{2}}$ ein Wert angenommen, so kann unter zusätzlicher Verwendung von

$$
P_{\mathrm{CO}_{2}}+P_{\mathrm{O}_{2}}+P_{\mathrm{N}_{2}}=B-47
$$

die stationäre Zusammensetzung des Atelektase-Gases berechnet werden.

$P^{\prime} \mathrm{O}_{2}-{ }^{0} P_{\mathrm{O}_{2}}$ kann in folgender Weise durch das Verhältnis von Anfangsdruck-Differenz zu Enddruck-Differenz $\left(\Delta P_{0} / \Delta P^{\prime}\right)_{0_{2}}=q$ ausgedrückt werden.

$$
\begin{aligned}
P_{\mathrm{O}_{2}}^{\prime}-{ }^{0} P_{\mathrm{O}_{2}} & =P_{\mathrm{O}_{2}}-{ }^{0} P_{\mathrm{O}_{2}}-\left(\Delta P^{\prime}\right)_{\mathrm{O}_{2}}, \\
\left(\Delta P^{\prime}\right)_{\mathrm{O}_{2}} & =\frac{\left(\Delta P_{0}\right) \mathrm{O}_{2}}{q}, \\
P_{\mathrm{O}_{2}}^{\prime}-{ }^{0} P_{\mathrm{O}_{2}} & =P_{\mathrm{O}_{2}}-{ }^{0} P_{\mathrm{O}_{2}}-\frac{\left(\Delta P_{0}\right)_{\mathrm{O}_{2}}}{q} \\
\left(\Delta P_{0}\right)_{\mathrm{O}_{2}} & =P_{\mathrm{O}_{2}}-{ }^{0} P_{\mathrm{O}_{2}}, \\
P_{\mathrm{O}_{2}}^{\prime}-{ }^{0} P_{\mathrm{O}_{2}} & =\left(P_{\mathrm{O}_{2}}-{ }^{0} P_{\mathrm{O}_{2}}\right)[1-(1 / q)] .
\end{aligned}
$$

Setzt man dies in $(6 \mathrm{~b})$ ein und vereinfacht weiter, so erhält man:

$$
\begin{array}{r}
\beta_{\mathrm{CO}_{2}}\left(1-\frac{{ }^{0} P_{\mathrm{CO}_{2}}}{P_{\mathrm{CO}_{2}}}\right)=\beta_{\mathrm{O}_{2}}\left(1-\frac{1}{q}\right)\left(1-\frac{{ }^{0} P_{\mathrm{O}_{2}}}{P_{\mathrm{O}_{2}}}\right)= \\
=\beta_{\mathrm{N}_{2}}\left(1-\frac{{ }^{0} P_{\mathrm{N}_{2}}}{P_{\mathrm{N}_{2}}}\right)=v .
\end{array}
$$

Tab. 2 enthält die Berechnungen der stationären $\mathrm{Zu}$ sammensetzung des Atelektase-Gases mit Variation der Annahmen über $q$ und über $\beta_{\mathrm{N}_{2}}$, wobei die zugrunde gelegten Annahmen jeweils in den linken Spalten und die Ergebnisse der Rechnung in den rechten Spalten erscheinen. Durchweg wurden folgende Partialdrucke ${ }^{0} P_{\mathrm{i}}$ des venösen Mischbluts angenommen:

$$
{ }^{0} P_{\mathrm{CO}_{2}}=50 \text { Torr, }{ }^{0} P_{\mathrm{O}_{2}}=40 \text { Torr, }{ }^{0} P_{\mathrm{N}_{2}}=560 \text { Torr, }
$$

ferner ein Barometerstand von 747 Torr, also

$$
P_{\mathrm{CO}_{2}}+P_{\mathrm{O}_{2}}+P_{\mathrm{N}_{2}}=700 \text { Torr. }
$$

15 K. H. Gertz u. H. H. Loeschcke, Z. Naturforschg. 9 b, 1, [1954]. 


\begin{tabular}{|c|c|c|c|c|c|c|c|c|c|c|c|}
\hline \multirow{2}{*}{$\begin{array}{c}\left(\frac{\Delta P_{0}}{\Delta P^{\prime}}\right)_{\mathrm{O}_{2}} \\
=q\end{array}$} & \multirow[t]{2}{*}{$\operatorname{Gas}_{i}$} & \multirow{2}{*}{$\begin{array}{c}\beta_{\mathrm{i}} \\
{\left[\mathrm{at}^{-1}\right]}\end{array}$} & \multirow{2}{*}{$\begin{array}{c}\text { Stat. } \\
\text { Druck } \\
P_{\mathrm{i}}[\text { Torr }]\end{array}$} & \multicolumn{3}{|c|}{$\begin{array}{c}\text { Gasabsorptionsstrom } \\
\dot{V}_{\mathrm{i}}\left[\mathrm{ml} \mathrm{min}^{-1}\right]\end{array}$} & \multirow{2}{*}{ 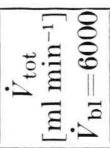 } & \multirow{2}{*}{$\begin{array}{c}\text { Mittl. } \\
\text { Druck- } \\
\text { Differenz } \\
\Delta P_{\mathrm{i}}[\text { Torr }]\end{array}$} & \multicolumn{3}{|c|}{$\begin{array}{c}\text { Diffusionsfaktor } \\
D F\left[\mathrm{ml} \mathrm{Torr}^{-1} \mathrm{~min}^{-1}\right]\end{array}$} \\
\hline & & & & $\dot{V}_{\mathrm{bl}}=4000$ & $\dot{V}_{\mathrm{bl}}=5000$ & $\dot{V}_{\mathrm{bl}}=6000$ & & & $\dot{V}_{\mathrm{bl}}=4000$ & $\dot{V}_{\mathrm{bl}}=500$ & $\dot{V}_{\mathrm{bl}}=6000$ \\
\hline 2 & $\begin{array}{r}\mathrm{CO}_{2} \\
\mathrm{O}_{2} \\
\mathrm{~N}_{2}\end{array}$ & $\begin{array}{l}2,3 \\
1,5 \\
0,012\end{array}$ & $\begin{array}{r}50,02 \\
40,05 \\
609,93\end{array}$ & & & & 5,4 & $\begin{array}{l}0,002 \\
0,04 \\
1,24\end{array}$ & $\begin{array}{r}130 \\
5,8 \\
2,5\end{array}$ & $\begin{array}{r}160 \\
7,2 \\
3,2\end{array}$ & $\begin{array}{r}200 \\
8,6 \\
3,8\end{array}$ \\
\hline 2 & $\begin{array}{r}\mathrm{CO}_{2} \\
\mathrm{O}_{2} \\
\mathrm{~N}_{2}^{2}\end{array}$ & $\begin{array}{l}2,3 \\
1,5 \\
0,013\end{array}$ & $\begin{array}{r}50,02 \\
40,06 \\
609,92\end{array}$ & & & 0,420 & 5,9 & $\begin{array}{l}0,002 \\
0,04 \\
1,5\end{array}$ & $\begin{array}{r}140 \\
5,5 \\
2,3\end{array}$ & $\begin{array}{r}180 \\
6,8 \\
2,8\end{array}$ & $\begin{array}{r}190 \\
8,5 \\
3,4\end{array}$ \\
\hline 2 & $\begin{array}{r}\mathrm{CO}_{2} \\
\mathrm{O}_{2} \\
\mathrm{~N}_{2}\end{array}$ & $\begin{array}{l}2,3 \\
1,5 \\
0,02\end{array}$ & $\begin{array}{r}50,04 \\
40,10 \\
609,86\end{array}$ & & & $\begin{array}{l}0,638 \\
0,510 \\
7,78\end{array}$ & 8,9 & $\begin{array}{l}0,004 \\
0,07 \\
2,05\end{array}$ & $\begin{array}{c}100 \\
5,0 \\
2,5\end{array}$ & $\begin{array}{r}130 \\
6,2 \\
3,1\end{array}$ & $\begin{array}{r}170 \\
7,1 \\
3,8\end{array}$ \\
\hline 3 & $\begin{array}{r}\mathrm{CO}_{2} \\
\mathrm{O}_{2} \\
\mathrm{~N}_{2}\end{array}$ & $\begin{array}{l}2,3 \\
1,5 \\
0,012\end{array}$ & $\begin{array}{r}50,02 \\
40,04 \\
609,94\end{array}$ & & & 4,72 & 5,4 & $\begin{array}{l}0,001 \\
0,02 \\
0,78\end{array}$ & $\begin{array}{c}220 \\
8,6 \\
4,0\end{array}$ & $\begin{array}{r}270 \\
10,8 \\
5,1\end{array}$ & $\begin{array}{r}320 \\
13,0 \\
6,1\end{array}$ \\
\hline 3 & $\begin{array}{r}\mathrm{CO}_{2} \\
\mathrm{O}_{2} \\
\mathrm{~N}_{2}\end{array}$ & $\begin{array}{l}2,3 \\
1,5 \\
0,02\end{array}$ & $\begin{array}{r}50,04 \\
40,10 \\
609,86\end{array}$ & & & 7,90 & 9,1 & $\begin{array}{l}0,002 \\
0,04 \\
1,29\end{array}$ & $\begin{array}{r}180 \\
8,7 \\
4,1\end{array}$ & $\begin{array}{r}220 \\
10,8 \\
5,1\end{array}$ & $\begin{array}{r}270 \\
13,0 \\
6,1\end{array}$ \\
\hline 10 & $\begin{array}{r}\mathrm{CO}_{2} \\
\mathrm{O}_{2} \\
\mathrm{~N}_{2}\end{array}$ & $\begin{array}{l}2,3 \\
1,5 \\
0,012\end{array}$ & $\begin{array}{r}50,02 \\
40,03 \\
609,95\end{array}$ & $\begin{array}{l}0,258 \\
0,207 \\
3,15\end{array}$ & $\begin{array}{l}0,323 \\
0,258 \\
3,94\end{array}$ & $\begin{array}{l}0,310 \\
4,72\end{array}$ & 5,4 & $\begin{array}{l}0,001 \\
0,01 \\
0,37\end{array}$ & $\begin{array}{r}430 \\
17,5 \\
8,5\end{array}$ & $\begin{array}{c}540 \\
21,5 \\
10,6\end{array}$ & $\begin{array}{c}650 \\
25,8 \\
24,8\end{array}$ \\
\hline 100 & $\begin{array}{r}\mathrm{CO}_{2} \\
\mathrm{O}_{2} \\
\mathrm{~N}_{2}\end{array}$ & $\begin{array}{l}2,3 \\
1,5 \\
0,012\end{array}$ & $\begin{array}{r}50,02 \\
40,03 \\
609,95\end{array}$ & $\begin{array}{l}0,258 \\
0,207 \\
3,16\end{array}$ & $\begin{array}{l}0,324 \\
0,258 \\
3,94\end{array}$ & $\begin{array}{l}0,388 \\
0,310 \\
4,72\end{array}$ & 5,4 & $\begin{array}{l}0,0003 \\
0,006 \\
0,19\end{array}$ & $\begin{array}{r}860 \\
37 \\
17\end{array}$ & $\begin{array}{r}1080 \\
46 \\
21\end{array}$ & $\begin{array}{r}1290 \\
55 \\
25\end{array}$ \\
\hline 3 & $\begin{array}{r}\mathrm{CO}_{2} \\
\mathrm{O}_{2} \\
\mathrm{~N}_{2}\end{array}$ & $\begin{array}{l}2,3 \\
2,0 \\
0,012\end{array}$ & $\begin{array}{r}50,02 \\
40,03 \\
609,95\end{array}$ & $\begin{array}{l}0,258 \\
0,207 \\
3,15\end{array}$ & $\begin{array}{l}0,323 \\
0,258 \\
3,94\end{array}$ & $\begin{array}{l}0,388 \\
0,310 \\
4,72\end{array}$ & 5,4 & $\begin{array}{l}0,0009 \\
0,06 \\
0,58\end{array}$ & $\begin{array}{r}270 \\
3,8 \\
5,4\end{array}$ & $\begin{array}{r}370 \\
4,7 \\
6,8\end{array}$ & $\begin{array}{r}430 \\
5,7 \\
8,2\end{array}$ \\
\hline
\end{tabular}

Tab. 2. Berechnung der Gasdrucke $P_{\mathrm{i}}$ und der mittleren Partialdruck-Differenzen der einzelnen Gase $\overline{\Delta P_{\mathrm{i}}}$ im stationären Zustand im Atelektase-Gas, ferner der Absorptionsströme der einzelnen Gase $\dot{V}_{\mathrm{i}}$ und der Diffusionsfaktoren $D F_{\mathrm{i}}$ für HerzminutenVolumina von 4000,5000 und $6000 \mathrm{l} \cdot \mathrm{min}^{-1}$ sowie des Gesamtgasstromes $\dot{V}$ tot im stationären Zustand, alles für verschiedene Werte des Verhältnisses der Anfangs-Druckdifferenz zur End-Druckdifferenz $\left(\Delta P_{0} / \Delta P^{\prime}\right)_{0_{2}}=q$ und der Löslichkeit von Stickstoff $\beta_{\mathrm{N}_{2}}$, bzw. der scheinbaren Löslichkeit von Sauerstoff $\beta_{\mathrm{O}_{2}}$. Die Gas-Partialdrucke des venösen Mischbluts wurden wie folgt angenommen: ${ }^{0} \mathrm{P}_{\mathrm{CO}_{2}}=50$ Torr, ${ }^{0} \mathrm{P}_{\mathrm{O}_{2}}=40$ Torr, ${ }^{0} \mathrm{P}_{\mathrm{N}_{2}}=560$ Torr. Barometerdruck $-47=700$ Torr. In den Spalten 1 und 3 sind die jeweils veränderten Variablen durch Kursivdruck kenntlich gemacht.

\section{Berechnung der mitttleren Partialdruck-Differenzen zwischen Gas- und Blutphase}

Durch die Berechnung der Gas-Partialdrucke $P_{\mathrm{i}}$ im Atelektase-Gas sind auch die Ausgangs-Differenzen zwischen Gasphase und stromaufwärtigem Ende der Kapillare bekannt als $P_{\mathrm{i}}-{ }^{0} P_{\mathrm{i}}=\left(\Delta P_{0}\right)_{\mathrm{i}}$. Die mitttlere Differenz $(\overline{\Delta P})_{O_{2}}$ wird nach $\mathrm{Gl} .(9 \mathrm{~b})$ in der folgenden umgewandelten Form berechnet:

$$
\overline{\Delta P}_{\mathrm{O}_{2}}=\frac{\left(\Delta P_{0}\right)_{0_{2}}[1-(1 / q)]}{\ln q} .
$$

Für $\mathrm{CO}_{2}$ und $\mathrm{N}_{2}$ vereinfacht sich die Formel zu

$$
\overline{\Delta P}_{\mathrm{CO}_{2}}=\frac{\left(\Delta P_{0}\right) \mathrm{CO}_{2}}{\ln \left(\Delta P_{0} / \Delta P^{\prime}\right) \mathrm{CO}_{2}} \text { und } \overline{\Delta P}_{\mathrm{N}_{2}}=\frac{\left(\Delta P_{0}\right)_{\mathrm{N}_{2}}}{\ln \left(\Delta P_{0} / \Delta P^{\prime}\right) \mathrm{N}_{2}} .
$$

Für $\ln \left(\Delta P_{0} / \Delta P^{\prime}\right)_{i}$ werden die oben berechneten bzw. bei abweichenden Annahmen die entsprechend berechneten Zahlenwerte eingesetzt. Die so erhaltenen $\Delta P_{\mathrm{i}}$ sind in Tab. 2 aufgenommen. Sie sind sämtlich mit den schon erwähnten und der Größe nach unbekannten Feh- lern der Integration der Sauerstoffdruck-Zunahme über die Austauschstrecke behaftet.

\section{Berechnung der Diffusionsströme}

Die Diffusionsströme erhält man einfach aus der Beziehung

$$
\left.\dot{V}_{\mathrm{i}}=v \dot{V}_{\mathrm{bl}} \cdot P_{\mathrm{i}}, \quad \text { [vgl. }(6 \mathrm{a})\right]
$$

wobei für die in die Tabelle aufgenommenen Werte die Herzminuten-Volumina $\dot{V}_{\mathrm{bl}}$ von 4000,5000 und $6000 \mathrm{ml} \mathrm{m^{-1 }}$ eingesetzt wurden. Diese Werte sind Vergleichswerte und für eine bestimmte Atelektase müßte die Durchblutung nur des atelektasierenden Gebietes eingesetzt werden. Berechnung für das gesamte Herzminuten-Volumen ist aber zweckmäßig, da sie erlaubt, durch Division des Sauerstoffstroms durch die mittlere Partialdruck-Differenz den Diffusionsfaktor (diffusion capacity) für diese Lunge zu berechnen. Ferner kann der so erhaltene Gesamtgasstrom verglichen werden mit den Versuchen von $D_{\text {ALE }}$ und $R_{A H N}{ }^{5}$, in denen experimentell die Geschwindigkeit der Absorption des Gases einer rückatmenden Lunge bestimmt wurde. 


\section{Berechnung des Diffusionsfaktors (diffusion capacity)}

Aus Diffusionsstrom und mittlerer Partialdruck-Differenz ergibt sich der Diffusionsfaktor, der abhängig ist von der für die Berechnung der Diffusionsströme eingesetzten Durchblutung.

\section{Diskussion}

Für die Berechnung der stationären Zusammensetzung des Atelektase-Gases wurde nur das Ficksche Prinzip verwendet und ein Zahlenwert für das Verhältnis von Anfangs-Partialdruck-Differenz zu End-Partialdruck-Differenz des Sauerstoffs $\left(\Delta P_{0} / \Delta P^{\prime}\right)_{0_{2}}=q$ zwischen Alveolarraum und Blutgefäß eingesetzt und schließlich ein Zahlenwert für die Abhängigkeit der $\mathrm{O}_{2}$-Bindung vom $\mathrm{O}_{2}$-Druck eingesetzt. Das Diffusionsgesetz wurde nur verwendet, um Unvollständigkeit des Partialdruck-Ausgleichs zwischen Alveolarraum und Blutgefäß für $\mathrm{CO}_{2}$ und $\mathrm{N}_{2}$ auszuschließen. Variation von $q$ zwischen 2 und 100 hat keinen ins Gewicht fallenden Einfluß auf die Höhe des $\mathrm{O}_{2}$-Druckes im AtelektaseGas, der in der gesamten Tabelle nur zwischen 40,03 und 40,10 Torr variiert. Es kann festgestellt werden, daß der $\mathrm{O}_{2}$-Druck im Atelektase-Gebiet in jedem Fall dem $\mathrm{O}_{2}$-Druck des venösen Mischbluts sehr nahe liegt. Er dürfte ihn bei Luftatmung kaum um mehr als 0,1 Torr überschreiten. Größer als die relative Schwankung der absoluten $\mathrm{O}_{2}$-Drucke ist die relative Schwankung der Differenzen gegenüber dem $\mathrm{O}_{2}$-Druck des venösen Mischbluts bei Änderung von $q$. Das führt dazu, daß Änderung von $q$ einen Einfluß auf den Diffusionsfaktor hat.

Vergleicht man die stationäre Zusammensetzung des für Atelektase-Gas berechneten „zweiten“ Modells, d. h. der von Blutgefäßen umgebenen Gastasche, mit der im einfacheren „Grenzschichtmodell“, so stimmen beide darin überein, daß sie dem Stickstoff die führende (oder besser bremsende) Rolle bei der Absorption zusprechen. Im zweiten Modell ist die Angleichung der Drucke von $\mathrm{CO}_{2}$ und $\mathrm{O}_{2}$ aber noch weitgehender als im Grenzschichtmodell.

Für die Berechnung der Diffusionsströme im Zustand konstanter Zusammensetzung wurden ebenfalls die Diffusionsgesetze noch nicht verwendet. Die Diffusionsströme der einzelnen Gase erweisen sich im berechneten Bereich als nicht abhängig von

16 F. J. W. Roughton u. R. F. Forster, J. appl. Physiol. 11, 290 [1957].
$\left(\Delta P_{0} / \Delta P^{\prime}\right)_{O_{2}}=q$. Auch eine Änderung der $\mathrm{O}_{2}$ Bindungsfähigkeit $\beta_{02}$ hat keinen erkennbaren Einfluß. Hingegen ist ein starker Einfluß der Bindungsfähigkeit für $\mathrm{N}_{2} \quad \beta_{\mathrm{N}_{2}}$ sichtbar. Dies rührt daher, daß bei den gegebenen Zahlenwerten die für die Berechnung der Diffusionsströme verwendete Größe $v$ nur sehr wenig von $\beta_{\mathrm{O}_{2}}$ und $\beta_{\mathrm{CO}_{2}}$, dagegen stark von $\beta_{\mathrm{N}_{2}}$ abhängig ist, was nicht anschaulich erklärt werden kann. Die Bindungsfähigkeit für Stickstoff $\beta_{\mathrm{N}_{2}}$ ist gleich der Löslichkeit $\alpha_{\mathrm{N}_{2}}$. In den Ableitungen wurde trotzdem zwischen $\beta_{\mathrm{N}_{2}}$ und $\alpha_{\mathrm{N}_{2}}$ unterschieden. Die beiden Ausdrücke beziehen sich hier auf verschiedene Phasen, $\beta_{\mathrm{N}_{2}}$ ist die Löslichkeit im Blut, und $\alpha_{\mathrm{N}_{2}}$ stellt die Löslichkeit in der trennenden Membran dar. Roughton und Forster ${ }^{16,17}$ haben neuerdings klargestellt, daß die Diffusionsschicht in 2 Phasen zu unterteilen ist, nämlich Membran und Blut. Dem entspricht die hier zunächst durchgeführte Unterscheidung zwischen $\alpha_{\mathrm{N}_{2}}$ und $\beta_{\mathrm{N}_{2}}$. Diese Unterscheidung wurde aus Gründen der Vereinfachung in der Rechnung fallen gelassen. Der korrekte Wert für $\beta_{\mathrm{N}_{2}}$ sollte 0,013 sein. Man erhält mit diesem Wert deutlich höhere Diffusionsströme als mit 0,012. Eine Übertreibung dieses Einflusses erhält man bei Verwendung von $\beta_{\mathrm{N}_{2}}=0,02$, einem Wert, der auf jeden Fall zu hoch gegriffen ist.

Der Gesamt-Diffusionsstrom aller drei Gase zusammen, der in den letzten Spalten der Tab. 2 aufgenommen wurde, erlaubt einen Vergleich mit den experimentellen Daten von DaLE und RAHN ${ }^{5}$. Diese Autoren haben u. a. bei Luftatmung die eine Lunge von Hunden durch Bronchialkatheter isoliert und mit einem bestimmten Ausgangsvolumen rückatmen lassen. Der mittlere Gas-Absorptionsstrom einer Lunge bei Hunden mit einem mittleren Gewicht von $20 \mathrm{~kg}$ betrug $0,92 \mathrm{ml} \mathrm{min}^{-1}$. Für zwei Lungen würde der Strom 1,84 $\mathrm{ml} \mathrm{min}^{-1}$ sein. Berechnet man den Absorptionsstrom auf ein Gewicht von $60 \mathrm{~kg}$ unter der Annahme, daß das Herzminuten-Volumen, welches die Größe ist, auf die es ankommt, nicht linear mit dem Gewicht, sondern mit dem Gewicht ${ }^{3 / 4}$ zunimmt, im gegebenen Falle also einem Faktor von 2,28, so erhält man einen Absorptionsstrom von $4,2 \mathrm{ml} \mathrm{min}{ }^{-1}$. Weiterhin betrug die PartialdruckDifferenz des Stickstoffs wahrscheinlich 20 und nicht wie bei den Rechnungen der Tab. 250 Torr (wobei angenommen ist, daß in den Versuchen von DALE und RAHN beide Lungen gleich durchblutet waren), so

17 R. F. Forster, F. J. W. Roughton, L. Cander, W. A. Briscoe u. F. Kreuzer, J. appl. Physiol. 11, 277 [1957]. 
würde man einen Absorptionsstrom von 4,2 $\cdot 50 / 20$ erhalten, also etwa 10,5 $\mathrm{ml} \mathrm{min}^{-1}$. Dieser Wert wäre mit aller Reserve mit den Ergebnissen der Berechnung in Tab. 2 zu vergleichen. Werte dieser Höhe werden in der Tabelle in keinem der berechneten Beispiele ganz erreicht, wenn man die Rechnungen mit $\beta_{\mathrm{X}_{2}}=0,02$ als unwirklich ausschließt. Immerhin kommen die Werte dann, wenn man hohe HerzminutenVolumina einsetzt, den experimentellen Werten ziemlich nahe. Auch ist zu erwarten, daß die HerzminutenVolumina in den Versuchen von DALE und RAHN hoch waren, da der arterielle $\mathrm{O}_{2}$-Druck bedeutend erniedrigt war. Auch die Analysen der AtelektaseLuft von DALE und $\mathrm{RAHN}^{5}$ zeigen ganz deutlich, daß $\mathrm{O}_{2}$-Druck und $\mathrm{CO}_{2}$-Druck nicht weit von den Drucken dieser Gase im Blut entfernt sind, und die Druckdifferenz nahezu ausschließlich vom Stickstoff getragen wird. Rechnung und Experiment sind in diesem Punkt in völlig genügender Übereinstimmung. Die Rechnung scheint in diesem Punkte dem Experiment überlegen zu sein, da sie zeigt, daß die PartialdruckDifferenzen von $\mathrm{O}_{2}$ und $\mathrm{CO}_{2}$ kleiner sein müssen, als selbst die besten analytischen Methoden im Augenblick feststellen können.

Wichtig ist festzuhalten, daß die Größe des Absorptionsstroms, der im wesentlichen ein Stickstoffstrom ist, fast ausschließlich begrenzt ist durch die Transportfähigkeit des Blutes für $\mathrm{N}_{2}$, also durch Herzminuten-Volumen und Löslichkeit von $\mathrm{N}_{2}$ im den.

Die Berechnung der mittleren Partialdruck-Differenzen der einzelnen Gase in der durchgeführten Weise ist mit großen Fehlern behaftet. Der wirkliche Vorgang ist bedeutend komplizierter, da das Gas vom Alveolarraum her zunächst die Trennwand zwischen Alveole und Blut zu passieren hat, dann ins Plasma diffundiert, in der Erythrocyte:ımembran auf ein Hindernis stößt und sich schließlich im Erythrocyten selbst ausbreitet. Das sind vier verschiedene Phasen und eine Berechnung dieses Problems unter Verwendung einer Abklingfunktion wäre für $\mathrm{N}_{2}$ allein zulässig, für $\mathrm{CO}_{2}$, dessen Bindungskurve im in Frage kommenden Bereich genügend linear ist, wäre die Approximation für den untersuchten Fall der Atelektase-Bildung gut, wenn nicht eine Wechselwirkung mit der $\mathrm{O}_{2}$-Bindung $(\mathrm{H}$ ald a n e$\mathrm{C}$ hristiansen-Effekt) aufträte. Für $\mathrm{O}_{2}$ gilt das gleiche (B o h r - Effekt). Im Gegensatz zum norma-

${ }^{18}$ F. Kreuzer, Helv. physiol. pharmacol. Acta 8, 505 [1950]. len Gasaustausch ist bei der Absorption des Atelektase-Gases die Richtung des $\mathrm{CO}_{2}$-Stromes und des $\mathrm{O}_{2}$-Stromes übereinstimmend. Die durch die Wechselwirkung beider Gase bedingte Abweichung ist darum nicht einfach zu übersehen, weil $\mathrm{CO}_{2}$ zweifellos zunächst sehr viel schneller in das Blut einströmt als $\mathrm{O}_{2}$. Der $\mathrm{O}_{2}$-Druck im Blut wird dadurch angehoben. Dies dürfte sich so auswirken, daß die berechneten mittleren Partialdruck-Differenzen $\overline{\Delta P}$ für $\mathrm{O}_{2}$ höher erscheinen als die wirklichen. Einen Anhaltspunkt dafür, daß das wirklich so ist, hat man in den berechneten Diffusionsfaktoren (diffusion capacity), die durchweg niedriger als normal herauskommen, wenn man einen 50-proz. Druckausgleich $(q=2)$ des $\mathrm{O}_{2}$ ansetzt. Einen normalen Wert für den Diffusionsfaktor des $\mathrm{O}_{2}$ von $21,5 \mathrm{ml} \mathrm{Torr}^{-1} \mathrm{~min}^{-1} \mathrm{er}$ hält man erst bei Annahme eines 90-proz. Druckausgleichs $(q=10)$ für den Sauerstoff. Dieser Wert erscheint ein wenig zu hoch, da der Druckausgleich im steilen Teil der $\mathrm{O}_{2}$-Bindungskurve stattfindet. In jedem Fall liegen die berechneten Diffusionsfaktoren in der Nähe der besten auf andere Art bestimmten Werte $\left(\right.$ Thews ${ }^{9}$ ). Hierin kann eine Bestätigung der Brauchbarkeit der angewendeten Approximationen und der Richtigkeit der Überlegungen gesehen werden.

Die Wahl der Zahlenwerte für $\alpha_{\mathrm{i}}$ und $D_{\mathrm{i}}$ ging davon aus, daß diese immer nur als Quotient, z. B. $\alpha_{\mathrm{CO}_{2}} D_{\mathrm{CO} 2} / \alpha_{\mathrm{O}_{2}} D_{\mathrm{O}_{2}}$ [Gl. (12 b)], auftreten. Die absolute Höhe der Werte von $\alpha_{\mathrm{i}}$ und $D_{\mathrm{i}}$ geht also in die Rechnung gar nicht ein. Darum erschien es zweckmäßig, die Werte für Wasser einzusetzen in der Annahme, daß die Beeinflussung sowohl von $\alpha$ wie von $D$ durch den Eiweißgehalt (Kreuzer ${ }^{18}$, Thews ${ }^{19}$ ) für alle Gase übereinstimmt. Die Werte von Gertz und Loeschcke ${ }^{15}$ für $D$ wurden als Verhältniszahlen bestimmt und erfüllen darum die Forderung möglicherweise am besten.

Fragt man nun, wie sich die Gasabsorption in anderen geschlossenen Gastaschen verhalten dürfte, so läßt sich sagen, daß deren Verhalten grundsätzlich zwischen den beiden Extremen liegen muß, die durch das Beispiel der Atelektase mit für den Gasaustausch extrem günstiger Durchblutung einerseits und durch das „Grenzschicht-Modell“, bei dem die Diffusion durch eine nicht durchblutete Grenzschicht hindurch stattfinden muß, andererseits gegeben sind. Begrenzend für die Absorptions-Geschwindigkeit ist

19 G. Thews, Pflügers Arch. ges. Physiol. Menschen, Tiere 265, 138 [1957]. 
im erstgenannten Falle die Durchblutung und im zweiten die Diffusion durch die Grenzschicht. Vergleicht man das Grenzschicht-Modell und das die Durchblutung berücksichtigende Modell vom forma- len Standpunkt, so zeigt sich, daß das GrenzschichtModell derjenige Grenzfall des die Durchblutung berücksichtigenden Modells ist, bei dem $\overline{\Delta P}=\Delta P_{0}$ wird. Das zweite Modell ist also das allgemeinere

\title{
The ecdysial membrane of the moth, Ephestia kühniella $Z$.
}

\author{
By A. Glenn Richards * \\ Aus dem Max-Planck-Institut für Biologie, Abt. Küнs, Tübingen \\ (Z. Naturforschg. 13 b, 811-812 [1958] ; eingegangen am 15. August 1958)
}

\begin{abstract}
Histologische Untersuchungen an der Mehlmotte Ephestia kühniella stützen die Ansicht von MaLeK, daß die Ecdysialmembran der umgeformte innerste Teil der alten Cuticula ist. Die Folgerungen dieser Feststellung hinsichtlich des Sklerotisierungs-Prozesses werden kurz besprochen.
\end{abstract}

A few years ago, Passonneau and Williams ${ }^{1}$ described a new membrane found between the old and new cuticles of molting caterpillars and pupae of the moths Telea and Platysamia. They named it the "ecdysial membrane." Excellent photographs from serial sections are given by LowER ${ }^{2}$. A more detailed study of the membrane's chemical composition and ultrastructure by electron microscopy showed it to be a typical sheet of cuticle with recognizable outer, middle and inner sublayers, and hence led to the suggestion that it might possibly represent a distinct cuticle with the function of somehow facilitating the process of moulting ${ }^{3}$. More recently, $\mathrm{MA}_{\mathrm{A}}$ LEK $^{4}$ studied this membrane in locusts and presented evidence for suggesting that the membrane is not a separate cuticle but only the innermost lamina of the old cuticle modified by leakage into it (or them) of sclerotization compounds produced for the new cuticle. MALEK's suggestion is an attractive one, and I have come to think it is probably correct.

In the course of studying another problem I happened to obtain a specimen of the moth Ephestia kühniella that only partially completed the larvalpupal transformation. This specimen showed almost complete digestion of all the larval procuticle, the unshed skin being little more than epicuticle except for the few sclerotized regions and certain small areas. It also had a strong ecdysial membrane (Fig. $\left.1^{*}\right)$. And, interestingly, this ecdysial membrane became continuous with the inner part of the larval endocuticle at those places where for some reason the endocuticle had not been fully digested in this abnormal moult (Fig. 2) .

The above agrees with normal prepupae of Ephestia which show a somewhat different picture from Platysamia and Persectania. In the latter two species the ecdysial membrane appears fully formed when the old cuticle becomes separated from the underlying cell layer, but in Ephestia the prepupal cuticle separates from the epidermis with the ecdysial membrane partly free and partly still continuous with the endocuticle of which it clearly forms the innermost laminae (Fig. 3). Separation of the ecdysial membrane does not become complete until after the enzymes of the moulting fluid digest the remainder of the endocuticle.

It has recently been learned that the "wild-type," "black," and presumably most mutant stocks of Ephestia fail to digest any of the pupal endocuticle whereas the mutant " $h e$ " does (as do almost all in-

\footnotetext{
* Fulbright Research Scholar and Guggenheim Fellow. Permanent address: Department of Entomology \& Economic Zoology, University of Minnesota, St. Paul 1, Minnesota, U.S.A.

1 J. V. Passonneau, and C. M. Williams, J. exper. Biology 30 , 545 [1953].
}

2 H. F. Lower, Zool. J. (Anat.) 76, 165 [1957].

3 A. G. Richards, J. Morphology 96, 537 [1955].

4 S. R. A. MaLeK, Nature [London] 178, 1185 [1956].

5 A. G. Richards, Z. Naturforschg. 13 b, 813 [1958].

* Fig. 1-4 siehe Bildtafel S. 812 a. 